\section{COVID-19: pulmonale Endothelialitis und systemische Koagulopathie}

Connors JM et al. COVID-19 and its implications for thrombosis and anticoagulation. Blood 2020; 135: 2033-2040. doi:10.1182/blood.202000 6000

Ackermann M et al. Pulmonary Vascular Endothelialitis, Thrombosis, and Angiogenesis in Covid-19. N Engl J Med 2020; 383: 120-128. doi:10.1056/ NEJMoa2015432

Fast täglich erreichen uns neue Daten zum SARS-CoV-2. Die Studie in Blood fasst die Datenlage zu thromboembolischen Komplikationen und deren Management zusammen. Die histopathologische Untersuchung von Ackermann et al. zeigt pulmonale Besonderheiten bei Patienten, die an COVID-19 starben. Neben Thrombosen und Mikroangiopathie fand sich eine besondere Form der Angiogenese.

Ähnlich wie SARS-CoV gelangt das SARSCoV-2 über den ACE2-Rezeptor in die pulmonale Wirtszelle. Der Lungenbefall stellt als COVID-19 die Haupttodesursache der Infektion dar. Radiologisch finden sich periphere Milchglastrübungen, die bei schweren Verläufen die Berlin-Kriterien für ein akutes Lungenversagen ARDS erfüllen. Histologisch bestehen in frühen Stadien unspezifische Zeichen mit Alveolarschädigung, Ödemen und intraalveolären Fibrinablagerungen. Die Arbeitsgruppe um Ackermann stellt die morphologischen und molekularen Veränderungen in peripheren Lungenabschnitten von jeweils 7 Patienten vor, die an COVID-19 oder Influenza (Subtyp H1N1) starben. Als Kontrolle lagen Präparate von 10 Verstorbenen ohne Lungenkrankheit vor. Untersuchungsmethoden waren die Mikro-CT, histopathologische Untersuchung, Multiplex-Immunhistochemie, Elektronenmikroskopie, Corrosion-Cast-Technik und die direkte Multiplex-Gensequenzierung.

Die Lungen der Influenzagruppe waren signifikant schwerer als die der COVIDund Kontrollgruppe. Die Organe zeigten bei COVID-19 und Influenza eine diffuse Alveolarschädigung und infiltrierende perivaskuläre Lymphozyten. Verglichen mit den Kontrollen bestand bei COVID-19 und Influenza eine signifikant höhere ACE2-Expression von alveolären Epithelund Endothelzellen. Bei SARS-CoV-2-Infizierten fanden sich mehr CD4-positive und weniger CD8-positive T-Zellen sowie Neutrophile angrenzend an die epithelialen Liningzellen. 79 inflammationsassoziierte und 69 Angiogenesegene wiesen eine COVID-spezifische Regulation auf. Die Gefäßbefunde bei COVID-19 unterschieden sich zur Influenza in 3 Punkten distinkt:

- Endothelschädigung mit intrazellularem SARS-CoV-2 sowie zerstörten Endothelzellmembranen,

- verbreitete Thrombosen mit Mikroangiopathie und Verschluss der pulmonalen Kapillaren sowie

- signifikantes Gefäßwachstum durch intussuszeptive Angiogenese.

Mikrothrombi waren $9 \times$ häufiger nachweisbar und die intussuszeptive Angiogenese $2,7 \times$ ausgeprägter als bei der Influenza. Im Unterschied zum ebenfalls nachweisbaren Sprouting-Mechanismus kommt es bei der Intussuszeption zum Einfügen endothelialer Trennschichten, die bestehende Gefäße teilen.

\section{Management der Thrombosegefahr}

Connors und Ackermann stellen in ihrem Review fest, dass die mikrovaskuläre Endothelschädigung mit mikrozirkulatorischer Clot-Formation eine thrombotische Mikroangiopathie begünstigt. Darüber hinaus resultiert die Immunantwort des Wirts in einer gesteigerten Sekretion proinflammatorischer Zytokine mit pleiotropen Effekten, darunter eine Gerinnungsaktivierung, die über eine sepsisinduzierte Koagulopathie (SIC) bis zur disseminierten intravasalen Gerinnung (DIC) mit Verbrauchskoagulopathie führen kann. Bei COVID-19 finden sich als systemische Antwort auf die SARS-CoV-2-Infektion schon frühzeitig hämostaseologische Veränderungen. Mehrere internationale Studien belegten, dass Patienten mit initial erhöhten D-Dimeren ungünstigere Verläufe und eine gesteigerte Mortalität aufwiesen. Bei einem SIC-Score $\geq 4$ reduzierte eine Hepa- rintherapie die Mortalität um 24,2\%. In einer anderen Studie bestand bei D-Dimeren $\geq 3$ und prophylaktischem Heparin eine $20 \%$ geringere Sterblichkeit. In der frühen Infektionsphase waren PT, aPTT und die Thrombozyten nur minimal verändert.

Einige Patienten zeigten eine besonders starke inflammatorische Antwort im Sinne eines Zytokinsturms mit dramatischen Gerinnungsstörungen. Die Koagulopathie wird als Zeichen der Erkrankungsschwere und nicht als virusintrinsisch interpretiert. Hohe IL-6-Spiegel, eine Hyperfibrinogenämie und steigende D-Dimere korrelierten mit Krankheitsprogressionen. In schweren Fällen nahmen die D-Dimere ab dem 4. Tag zu und waren mit einer Sepsisentwicklung und längeren Krankenhausaufenthalten assoziiert. DIC traten 7-11 Tage nach dem Symptombeginn auf. Mikrovaskuläre Thrombosen könnten für ein Multiorganversagen bei prolongierten Infektionen verantwortlich sein.

COVID-assoziierte Gerinnungsstörungen inklusive DIC sollten behandelt werden, wie andere Koagulopathien bei anderen Ursachen, meinen Connors und Levy. Sie diskutieren:

- risikoabhängig ambulante Thromboembolieprophylaxe,

- Gerinnungsmonitoring mit D-Dimeren und Fibrinogen für alle stationären Patienten,

- Thromboembolieprophylaxe bei hospitalisierten Patienten,

- risikoabhängig eventuell intermediäre Heparindosis bei Intensivpatienten und ARDS,

- therapeutische Antikoagulation bei bestätigter VTE und vermuteter PE,

- Standardmanagement bei SIC,

- Standardmanagement bei DIC und bei Blutungen.

Eine gesteigerte prophylaktische Heparinisierung sei möglicherweise bei übergewichtigen Patienten und einer gesteigerten Prokoagulation sinnvoll. Weitere Belege stünden aus. Für Interaktionen von SARS-CoV-2 und Heparin liegen bislang keine Hinweise vor. Die Autoren raten von oralen Antikoagulanzien wegen des fehlenden Monitorings eher ab. 
FAZIT

Beide Studien weisen in die gleiche Richtung: Als Kernprobleme bei COVID-19 könnten sich die Gefäßveränderungen mit begünstigten Mikrothromben und die überschießende Gerinnung als Zeichen der Thromboinflammation erweisen. Dies habe auch zu einer Diskussion um gesteigerte oder therapeutische Heparindosen geführt. Obwohl D-Dimere, Sepsis und Verbrauchskoagulopathie mit der Mortalität korrelierten, reichen laut Connors und Ackermann die Daten nicht für die generelle Empfehlung einer therapeutischen Heparingabe aus. Ausnahmen seien natürlich Patienten mit anderen Indikationen, wie z. B. Vorhofflimmern, bestätigten Thrombosen oder besonderen Risikokonstellationen.

Dr. med. Susanne Krome, Melle 